PERSPECTIVE

\title{
Review of data and knowledge gaps regarding yellow fever vaccine-induced immunity and duration of protection
}

\author{
J. Erin Staples $\mathbb{D}^{1 凶}$, Alan D. T. Barrett ${ }^{2}$, Annelies Wilder-Smith ${ }^{3,4}$ and Joachim Hombach ${ }^{5}$
}

Yellow fever (YF) virus is a mosquito-borne flavivirus found in Sub-Saharan Africa and tropical South America. The virus causes YF, a viral hemorrhagic fever, which can be prevented by a live-attenuated vaccine, strain 17D. Despite the vaccine being very successful at decreasing disease risk, YF is considered a re-emerging disease due to the increased numbers of cases in the last 30 years. Until 2014, the vaccine was recommended to be administered with boosters every 10 years, but in 2014 the World Health Organization recommended removal of booster doses for all except special populations. This recommendation has been questioned and there have been reports of waning antibody titers in adults over time and more recently in pediatric populations. Clearly, the potential of waning antibody titers is a very important issue that needs to be carefully evaluated. In this Perspective, we review what is known about the correlate of protection for full-dose YF vaccine, current information on waning antibody titers, and gaps in knowledge. Overall, fundamental questions exist on the durability of protective immunity induced by YF vaccine, but interpretation of studies is complicated by the use of different assays and different cut-offs to measure seroprotective immunity, and differing results among certain endemic versus non-endemic populations. Notwithstanding the above, there are few well-characterized reports of vaccine failures, which one would expect to observe potentially more with the re-emergence of a severe disease. Overall, there is a need to improve YF disease surveillance, increase primary vaccination coverage rates in at-risk populations, and expand our understanding of the mechanism of protection of $Y F$ vaccine.

npj Vaccines (2020)5:54; https://doi.org/10.1038/s41541-020-0205-6

\section{INTRODUCTION}

Yellow fever (YF) virus, a mosquito-borne flavivirus, is present in tropical areas of Africa and South America. Infection in humans can produce a hemorrhagic fever and is fatal in $30-60 \%$ of persons with severe disease ${ }^{1,2}$. Recent decades have witnessed an unprecedented emergence of YF virus activity, including in highly urbanized areas where vaccination coverage was low ${ }^{3-5}$. It has been recently estimated that roughly 400 million individuals require vaccination within at-risk zones to potentially prevent epidemic of the disease though many more might be at risk due to the recent expansion of risk zones, particularly in Brazil ${ }^{3,6}$

YF vaccine was first developed in the 1930s after successful attenuation of the Asibi strain of $Y F$ virus to generate the strain $17 D^{7}$. Today, three substrains (17D-204, 17DD, and 17D-213) are used as vaccines and are manufactured by six companies, of which four are prequalified by the World Health Organization $(\mathrm{WHO})^{8}$. The vaccine is given as one dose either by subcutaneous or intramuscular administration, with $80 \%$ of vaccine recipients develop neutralizing antibodies 10 days post immunization and close to $100 \%$ by one month post immunization in clinical trials ${ }^{9}$. However, it has been noted that children $<2$ years of age can have lower seroconversion rates following a single dose of YF vaccine ${ }^{10}$. No human efficacy studies have ever been performed with the vaccine, but protection has been robustly demonstrated. Evidence for this conclusion include (1) reduction of laboratory-associated infections in vaccinated workers, (2) observation following initial use of the vaccine in Brazil and other South American countries that YF occurred only in unvaccinated persons, (3) rapid disappearance of cases during YF vaccination campaigns initiated during epidemics, (4) very few vaccine failures detected in any endemic country, and (5) protection of rhesus monkeys against virulent wild-type (WT) YF virus challenge by neutralizing antibodies generated in response to YF vaccination ${ }^{11-13}$.

A booster dose requirement for YF vaccine was first put into place in 1959 under the precursor to International Health Regulations (IHR), International Sanitary Regulations, with booster doses initially being required every 9 years based on available data ${ }^{14,15}$. The booster dose interval was changed in 1965 to every 10 years based on limited evidence from two published studies that showed neutralizing antibodies were present in most vaccine recipients, including those who received the vaccine in childhood, for at least 10 years after vaccination ${ }^{16,17}$. Starting in late 2011, the WHO Strategic Advisory Group of Experts (SAGE) on Immunization YF working group conducted a systematic review of $\sim 17$ unpublished and published studies that identified a very low number of vaccine failures and high seropositivity rates following vaccination over time ${ }^{18,19}$. From these additional, albeit observational data, SAGE concluded that a single primary dose of YF vaccine is sufficient to confer sustained immunity and lifelong protection against YF disease, and that a booster dose is not needed, except for special populations (e.g., immunocompromised and immunosuppressed $)^{20}$. In May 2014, the World Health Assembly adopted the recommendation to remove the 10-year booster dose requirement from the IHR, which was enacted in June $2016^{21}$. In 2014, the United States Advisory Committee on Immunization Practices (ACIP) YF vaccine working group conducted a similar systematic review of YF vaccine immunogenicity ${ }^{10}$. However, since SAGE's recommendation removed the IHR requirement for boosters, ACIP working group reviewed the available data to determine whether or not booster doses were

\footnotetext{
${ }^{1}$ Arboviral Diseases Branch, U.S. Centers for Disease Control and Prevention, Fort Collins, CO, USA. ${ }^{2}$ Department of Pathology and Sealy Institute for Vaccine Sciences, University of Texas Medical Branch, Galveston, TX, USA. ${ }^{3}$ Institute of Public Health, University of Heidelberg, Heidelberg, Germany. ${ }^{4}$ London School of Hygiene and Tropical Medicine, London, UK. ${ }^{5}$ World Health Organization, Geneva, Switzerland. ${ }^{凶}$ email: estaples@cdc.gov
} 
needed as ACIP had never recommended a booster dose of the vaccine before. Based on the available data, ACIP voted in 2015 that a single primary dose of YF vaccine provides long-lasting protection and is adequate for most travelers ${ }^{22}$. However, as a precautionary measure, it was noted that a booster dose may be given to travelers who received their last dose of $Y F$ vaccine at least 10 years previously and who will be in a higher-risk setting based on season, location, activities, and duration of their travel. This would include travelers who plan to spend a prolonged period in endemic areas or those traveling to highly endemic areas, such as rural West Africa during peak transmission season or an area with an ongoing outbreak.

Subsequent to SAGE and ACIP recommendations that a single dose of YF vaccine is sufficient to provide lifelong protection in most individuals, several have questioned this decision ${ }^{23-26}$. Furthermore, several recent studies have noted waning antibody titers after vaccination and potential vaccine failures ${ }^{27-34}$. Below we note what is known about vaccine immunity, review the additional data that have been generated using full-dose YF vaccine since the SAGE recommendation in 2013, and discuss next steps to determine if booster doses of YF vaccine are needed.

\section{WHAT CONSTITUTES YF VACCINE IMMUNITY?}

One of the key questions to know whether or not YF vaccine booster doses are needed is what constitutes protective vaccine immunity. The closest correlate of protection that exists for YF vaccination was established in one study of non-human primates vaccinated with YF vaccine and then challenged with virulent WT YF virus ${ }^{11}$. From this study, $\log _{10}$ neutralization index (LNI) of $\geq 0.7$ was established as a potential cut-off for protective immunity with 51 (94\%) of 54 surviving monkeys having a $L N I \geq 0.7$. In comparison, only one (8\%) of 12 monkeys who died when challenged had a LNI above 0.7. Currently, plaque reduction neutralization tests (PRNTs) are used to establish the quantitative titers of YF virus-specific antibodies as it uses less serum and is typically easier to perform. Current studies typically report either $90 \%$ PRNT (PRNT 90 ), PRNT $_{80}$, or PRNT $_{50}$ titers. Although a $\mathrm{PRNT}_{90}$ titer is more specific as it reduces the likelihood of positive results due to cross-reactive neutralizing antibodies from other flaviviruses, it measures at the bottom of the S-shaped neutralization curve, which leads to less variability and can lead to false-negative results for lower virus-specific antibody titers ${ }^{35}$. PRNT $_{50}$ titer are at the midpoint or more linear portion of an S-shaped curve making them higher, more variable and sensitive, but less specific. Most clinical trials for flavivirus vaccines use a PRNT $_{50}$ assay with a titer of 1 in 10 as a correlate of protection ${ }^{36-38}$. However, LNI and PRNT have never been formally compared using standardized reagents to understand how they might relate. Furthermore, it is unclear if neutralizing antibodies as measured using current assays are the only correlate of protection. Our understanding of the role of cellmediated immunity in both the initial immunologic response, as well as longer-term protection is advancing, but it also comes with the uncertainty of what might represent protective types and levels immunity that could prevent a person developing WT YF disease. However, there is general agreement that the pool of memory cells needs to be able to quickly proliferate when challenged to protect an individual as the incubation period of YF is typically short ranging from 3 to 6 days $24,39,40$.

The question of what constitutes vaccine immunologic memory is not unique to YF vaccine. Smallpox vaccine also was utilized before efficacy studies could be performed and the same questions about vaccine immunity are present for liveattenuated vaccines against vaccinia virus ${ }^{41}$. Although detection of antibodies is used to denote protective immunity following measles vaccination, it also has been documented that individuals lacking detectable neutralizing antibodies can develop secondary immune response with revaccination or exposure to measles virus suggesting that alternative types of immunity exist ${ }^{42}$.

Currently, whether or not the absence of detectable neutralizing antibodies represent an absence of protective immunity against WT YF disease is a critical knowledge gap for YF immunity. As noted above, it is also unclear what amount of antibody might be needed to protect someone against developing a symptomatic infection or viremia. Two studies have documented roughly onethird of individuals with preexisting YF virus-specific neutralizing antibodies fail to develop an anamnestic neutralizing antibody response (i.e., $\geq 4$-fold or greater increase in neutralization titers) following a booster dose suggesting sterilizing immunity that is correlated with higher pre-vaccination titers ${ }^{9,43}$. If it is correct that an absence of detectable neutralizing antibodies following primary immunization or the development of an amnestic response following a booster vaccine dose means an absence of protection for $\mathrm{YF}$ in a primary vaccinee, one might have expected more cases of WT YF disease to be reported in children 4-10 years post-vaccination ${ }^{33}$. However, epidemiologic data from the recent outbreaks in Brazil indicate that very few cases of WT disease occurred in children, with a lower incidence of WT disease in children compared to adults ${ }^{5,44}$. Although this might be secondary to who is being exposed or differences in clinical attack rate, the recent outbreaks occurring near and in urban areas as well as the notable occurrence of cases in women tend to suggest children were likely exposed to the virus in these recent outbreaks. Finally, the development of an amnestic response might not equate to a lack of protection, particularly if the kinetics of the immunologic response is fast enough to blunt the viremia due to a WT infection.

\section{VACCINE FAILURES}

Since 2013, there has been several reports of vaccine failures, one in peer-reviewed literature plus epidemiologic reports issued by public health authorities ${ }^{45-47}$. The published study, which has been cited by others in editorials and reviews to support the need for booster doses, came out in 2014 during the ACIP deliberations and describe individuals having a history of YF vaccination who later develop WT YF disease $24,26,45$. The ACIP YF vaccine working group contacted the Brazil Ministry of Health $(\mathrm{MOH})$ to verify that, as stated, 459 (55\%) of 831 YF cases in Brazil from 1973 to 2008 were vaccine failures, including $27(3 \%)$ primary vaccine failures (e.g., occurring after the first 10 days of vaccination but within the first 10 years of vaccination) and 432 (52\%) secondary vaccine failures (e.g., occurring more than 10 years after vaccination potentially due to waning antibody titers ${ }^{45}$. The Brazil $\mathrm{MOH}$ provided data to the working group noting that there were seven vaccine failures in Brazil from 1973 to 2008; five constituting primary vaccine failures, and two secondary vaccine failures occurring at 20 and 27 years post vaccination ${ }^{10,45,48-50}$. Unfortunately, there has never been a publication to clarify that the data were not accurate and it continues to be cited as evidence to support the need for booster doses ${ }^{33}$.

From data reported to the Pan American Health Organization (PAHO) during 2000-2014 and published on their website, 83 (7\%) of 1164 of sylvatic YF cases reported from Bolivia, Brazil, Colombia, and Peru occurred in individuals who reported receiving YF vaccine $^{46}$. More recently during the large outbreaks of YF in Brazil, an epidemiologic bulletin noted at least 11 cases of WT YF in individuals who were previously vaccinated and several more cases have been noted during a recent meeting ${ }^{47,51}$. Unfortunately, the information about these additional cases is very limited. It is unknown if these cases represent primary or secondary vaccine failures, whether and what confirmatory laboratory testing was performed, and the underlying medical history of the cases (e.g., immunosuppressed or compromised) that might have impacted their initial immunologic response to the vaccine or longer-term immunologic memory. Critically, given 
that YF IgM antibodies can persist for years following vaccination $^{52}$, obtaining information about how the diagnosis of WT YF disease was made is important to interpret these results. Furthermore, it is important to note that not all individuals respond to YF vaccination; there is a median seroconversion rate of $99 \%$ (range $81-100 \%$ ) in clinical trials ${ }^{8}$. Critically, for a state like Minas Gerais in Brazil with a population over 20 million, this means that even with $100 \%$ vaccination coverage more than 200,000 individuals who were vaccinated would fail to develop an immune response to the vaccine and would be at risk for developing disease if exposed.

\section{SEROPOSITIVITY IN VACCINATED INDIVIDUALS}

Since the SAGE recommendations in 2013, a number of articles have been published related to the immune response seen following YF vaccine, including cohorts of individuals in endemic and non-endemic locations, of different ages, and at different time points following vaccination. All studies used PRNT or microneutralization test for the detection of neutralizing antibodies against YF virus. However, the percent plaque reduction cut-off used and the definition of seropositivity or protection varied by study such that quantity of neutralizing antibodies measured in different studies are difficult to compare ${ }^{35}$. Furthermore, several of the studies did not use the international standard making comparison of seropositivity or antibody concentrations between studies further challenging ${ }^{53}$. The findings of these studies are summarized below.

\section{Humoral immunity in adults}

There are data on longer-term humoral immunity for at least eight distinct cohorts of adults in both YF endemic and non-endemic areas of the world who received a full dose of YF vaccine (Table $1)^{27,28,31,32,54-57}$. Notably, there were no apparent differences between studies undertaken in endemic and non-endemic countries. In the first 5 years post-vaccination, seropositivity in the cohorts was $>90 \%$. At $\geq 10$ years post-vaccination, the rates of seropositivity were generally lower ranging from $67 \%$ to $88 \%$ using PRNT $_{50}-$ PRNT $_{90}$, except for a small cohort of healthy volunteers in the Netherlands where 97\% (34/35) of individuals vaccinated with a full-dose of the vaccine were seropositive at 10 years when measured with $\mathrm{PRNT}_{80}{ }^{57}$. Interestingly, several of the studies saw higher rates of seropositivity 30-35 years postvaccination compared to rates at $10-20$ years post vaccination $^{54,56}$. However, the number of individuals in the later vaccination time points are quite limited and they likely received an older vaccination formulation, which have differing quantities of vaccine virus ${ }^{8}$, impacting the generalizability of these results. Several other factors likely impact the overall rates of seropositivity in these studies, such as (1) proof of vaccination ${ }^{27}$, (2) different seropositivity cut-offs ${ }^{28,32,35}$, (3) different individuals at each time point post-vaccination often with different demographic (e.g., age of vaccination) $)^{27,28,30,56}$, (4) potential natural boosting for residents and travelers to endemic areas, and (5) potentially receiving an additional doses of YF vaccine ${ }^{31}$.

\section{Humoral immunity in children}

There have been four additional published studies with short-term and long-term immunogenicity for children receiving a full dose of YF vaccine (Table 2). The published studies contain cohorts of children who received YF vaccination at 9-23 months of age. Of the two studies published evaluating the seroconversion rate following YF vaccination in children, the rates are highly variable within one of the studies and between the studies ${ }^{58,59}$. In a study of 595 children living in Colombia and Peru who received YF vaccine alone or with a tetravalent dengue vaccine on a YF vaccine backbone, the rate of seroconversion was noted to be
99.8-100\% when measured by $\mathrm{PRNT}_{50}$ and titer $\geq 10^{58}$. These rates were similar though slightly higher than the rates seen in Mali (95-98\%) among children who received a meningococcal A (Men A) vaccine either concurrently or serially with YF vaccine ${ }^{59}$. However, in the same Men A vaccine study, children in Ghana only achieved $68-79 \%$ seroconversion rates following $Y F$ vaccination. This same trend in lower rates of detectable antibodies between the two populations in the Men A study was seen when the cohorts were followed up at 2-6 years post-vaccination ${ }^{34}$. Seropositivity rates as low as $28 \%$ were reported for children in Ghana at 2.3 years post-vaccination, though the rate increased at 6 years post-vaccination to $43 \%$, compared to $50 \%$ seropositivity among the children in Mali at 4.5 years post-vaccination ${ }^{34}$. When demographic (age of vaccination, sex), vaccination and exposure history (season of vaccination and pre-vaccination titers), and nutritional status were compared between the children in Mali and Ghana, no significant differences were identified to explain the different rates of seropositivity between these two populations ${ }^{60}$. In the second study evaluating longer-term immunity in different cohorts of children in Brazil up to 10 years postvaccination, a substantial decline was noted in the seropositivity rates over time ${ }^{33}$. Using a titer $\geq 10$ with PRNT $_{50}, 54 \%$ of children were not seropositive at 7 years post-vaccination. Although the rates of seropositivity increased when using a lower titer cut-off (PRNT $\geq 5), 36 \%$ of children at 7 years post-vaccination lacked detectable neutralizing antibodies.

One potential explanation for the varying immune response both initially and potentially longer-term among the pediatric studies could be the age at which the children received their vaccine. Younger age groups might be expected to have a less robust initial immune response, potential immunologic interference from maternal antibodies, or more concomitant infections lead to a decreased immune response ${ }^{61,62}$. The cohorts in Mali, Ghana, and some of the children in the Brazil study received YF vaccine at 9 months of age. This is compared to children in Colombia and Peru who received the vaccine at 12 months of age and others in the Brazil cohort who were as old as 23 months when they were vaccinated. However, when the age of vaccination was assessed by the ACIP YF working group relative to the seroconversion rates, the analysis of results from aggregated studies found no difference in seroconversion rates when the children were vaccinated at 9 months of age compared to 12 months ${ }^{10,22}$.

With these new pediatric data, there are seemingly more questions than answers to the variability of the results between the pediatric cohorts. The authors of the studies and associated editorials question what contributes to the variability in results hypothesizing that it could be due to differences in immune microenvironment, vaccine substrains used, how the samples were handled, the test used, and potential difference in vaccine handling $33,61,63,64$. Furthermore, in both Ghana and Brazil, the authors questioned whether or not children had received another dose of the vaccine as the proportion seropositive was higher at later time points ${ }^{33,34}$.

\section{Additional immunogenicity data}

Since 2013, several studies have been published regarding cellular immunity, including CD8+, CD4+, and memory phenotypes, formed in response to YF vaccine ${ }^{30,54,55,65}$. However, the specific impact of alternative types of immunologic memory and their role in protecting persons against disease is not well-characterized or known.

\section{NEXT STEPS}

The studies published since SAGE and ACIP made their recommendation that one dose of $\mathrm{YF}$ vaccine is sufficient to 
J.E. Staples et al.

Table 1. Seropositivity following full-dose yellow fever (YF) vaccination in adults.

\begin{tabular}{|c|c|c|c|c|c|c|}
\hline $\begin{array}{l}\text { Kareko } 2019^{27} \\
\text { 17D-204 (USA) }\end{array}$ & Non-endemic & Obs & $\begin{array}{l}\text { PRNT }_{90} \\
\text { Titer } \geq 10\end{array}$ & $\begin{array}{l}1 \mathrm{~m}-61 \mathrm{y} \\
0-3 y^{a} \\
3-12 y \\
>12 y\end{array}$ & $\begin{array}{l}71 / 92 \\
12 / 13 \\
28 / 37 \\
22 / 32\end{array}$ & $\begin{array}{l}(77) \\
(92) \\
(76) \\
(67)\end{array}$ \\
\hline $\begin{array}{l}\text { Wieten } 2016^{\mathrm{b} 54} \\
\text { 17D-204 (Europe) }\end{array}$ & Non-endemic & Obs & $\begin{array}{l}\mathrm{PRNT}_{80} \\
\mathrm{IU} / \mathrm{mL} \geq 0.5\end{array}$ & $\begin{array}{l}11-40 y \\
35-40 y^{a}\end{array}$ & $\begin{array}{l}89 / 99 \\
6 / 6\end{array}$ & $\begin{array}{l}(90) \\
(100)\end{array}$ \\
\hline $\begin{array}{l}\text { Campi-Azevedo } 2019^{30} \\
\text { 17DD (Brazil) }\end{array}$ & Endemic & Obs & $\begin{array}{l}\text { Micro-PRNT } \\
\text { Titer }>50\end{array}$ & $\begin{array}{l}1 m-10+y \\
1 m \\
1-5 y \\
6-9 y \\
\geq 10 y\end{array}$ & $\begin{array}{l}155 / 178 \\
48 / 50 \\
38 / 40 \\
30 / 33 \\
39 / 55\end{array}$ & $\begin{array}{l}(87) \\
(96) \\
(95) \\
(90) \\
(71)\end{array}$ \\
\hline $\begin{array}{l}\text { Lindsey } 2018^{56} \\
\text { 17D-204 (USA) }\end{array}$ & Non-endemic & Obs & $\begin{array}{l}\text { PRNT }_{90} \\
\text { Titer }>10\end{array}$ & $\begin{array}{l}1 \mathrm{~m}-53 \mathrm{y} \\
0-4 y^{a} \\
5-9 y \\
10-19 y \\
20-29 y \\
\geq 30 y\end{array}$ & $\begin{array}{l}200 / 221 \\
128 / 136 \\
18 / 19 \\
34 / 42 \\
13 / 16 \\
7 / 8\end{array}$ & $\begin{array}{l}(90) \\
(94) \\
(95) \\
(81) \\
(81) \\
(88)\end{array}$ \\
\hline $\begin{array}{l}\text { Martins } 2018^{\mathrm{e} 32} \\
\text { 17DD (Brazil) }\end{array}$ & $\begin{array}{l}\text { Non-endemic } \\
\text { region of Brazil }\end{array}$ & Obs & $\begin{array}{l}\mathrm{PRNT}_{50} \\
\mathrm{mIU} / \mathrm{mL} \geq 2.7 \log _{10}\end{array}$ & $\begin{array}{l}1 \mathrm{~m} \\
10 \mathrm{~m} \\
8 \mathrm{y}\end{array}$ & $\begin{array}{l}128 / 131 \\
115 / 117 \\
56 / 68^{f}\end{array}$ & $\begin{array}{l}(97) \\
(98) \\
(82)\end{array}$ \\
\hline $\begin{array}{l}\text { Roukens } 2018^{57} \\
\text { 17D-204 (France) }\end{array}$ & Non-endemic & Obs & $\begin{array}{l}\mathrm{PRNT}_{80} \\
\text { Titer } \geq 10\end{array}$ & $10 y$ & $34 / 35$ & (97) \\
\hline
\end{tabular}

provide lifelong protection in most individuals provide additional data on YF vaccine immunity. Given the heterogeneity of results, in particular for the pediatric cohorts, further studies would be welcomed.

However, the basic questions that were debated in the discussions of both SAGE and ACIP still remain, how durable is the immunity elicited by YF vaccine and what constitutes protective immunity against YF virus infection and disease? To truly address these questions, additional research and data are needed. Increased transparency and sharing of information on potential vaccine failures are critical to better understand of the $>800$ million doses the vaccine that have been administered how many might have failed to provide both short-term and long-term protective immunity. With this is the need to continue improving and strengthening YF disease surveillance and laboratory testing ${ }^{66}$, not only to detect possible vaccine failures but also to obtain samples early enough to make a definitive diagnosis of WT disease by molecular testing. In addition, every effort must be made to ascertain the vaccination status of the patient. As noted above, using standards and evaluating the correlation between neutralization titers determined by LNI and PRNT would improve our ability to compare studies and begin to set thresholds as to what antibodies levels are needed to potentially prevent WT disease. Furthermore, additional research is needed to determine the kinetics of the immune response when a vaccinee receives a booster vaccine dose or has a WT infection (e.g., does an amnestic response mean a lack of adequate protection?) and to validate the immune correlate of protection following YF vaccination using more modern knowledge and techniques (e.g., assessing the role of cellular immunity). WHO currently plans to receive input from subject matter experts on how best to proceed with measuring YF vaccine immunity in a consistent manner to allow for comparability between studies.

Overall, we expect the debate of whether or not to give booster doses of YF vaccine to continue in lieu of more data. However, one clear public health action that can and should be taken now is to improve YF vaccination coverage among children living in at risk areas. Based on WHO and UNICEF estimates of vaccine coverage 


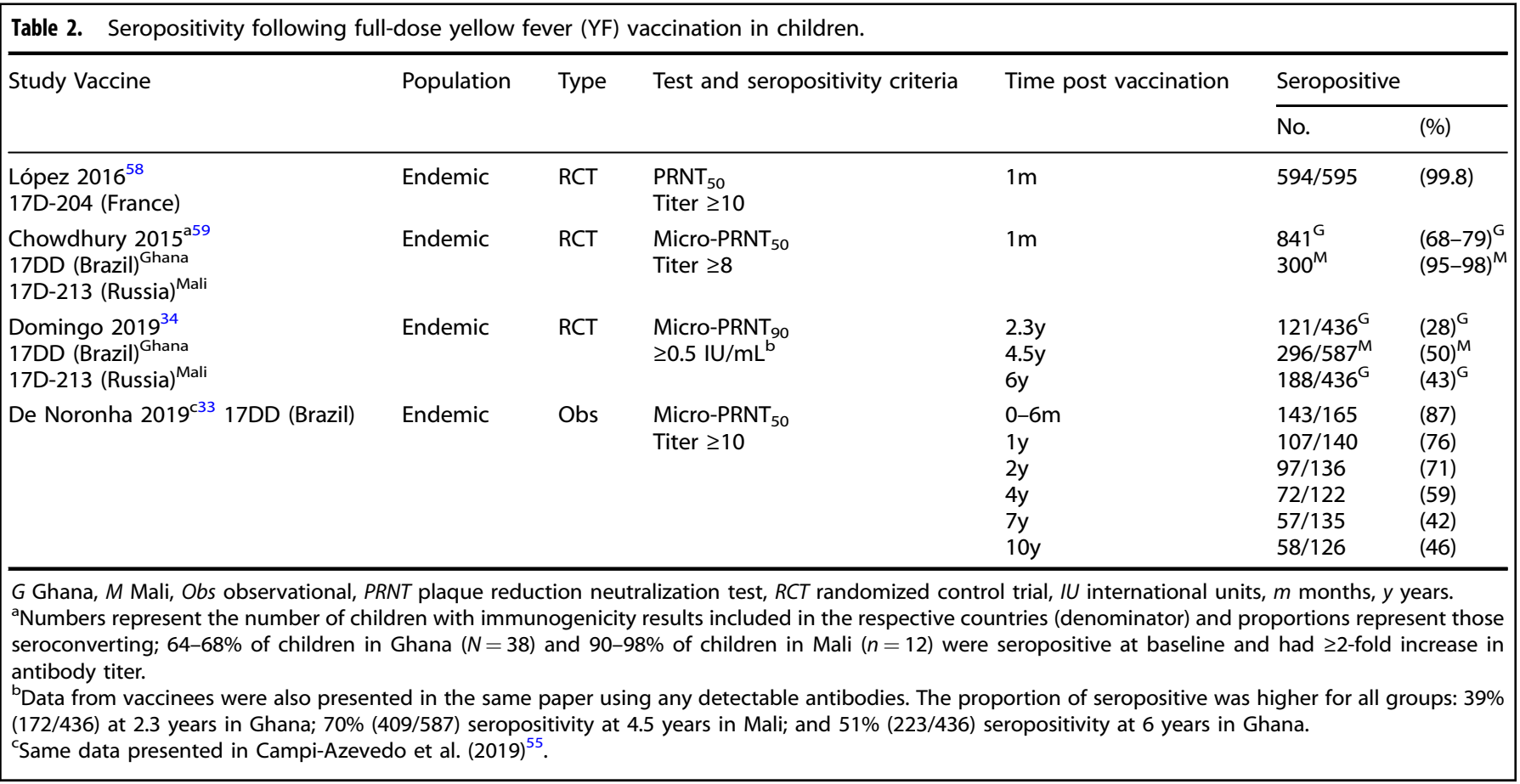

(WUENIC), YF vaccination rates among children living in YF endemic areas ranges from $42 \%$ to $97 \%$ (median of $85 \%$ ) in the Americas and $29-94 \%$ (median: $68 \%$ ) in Africa ${ }^{67}$. The current large outbreaks of measles throughout the world, including in YF endemic areas where the vaccines are often given at the same visit, reinforces poor YF vaccination rates that exist among children. If children do not even receive their first dose of YF vaccine, it is hard to focus on whether they might need a booster dose. We encourage researchers, clinicians, and public health officials to continue to evaluate and publish quality data on YF vaccine immunity and vaccine failures to inform public health policy related to YF vaccine use and optimize our ability to prevent YF.

Received: 13 January 2020; Accepted: 29 May 2020; Published online: 06 July 2020

\section{REFERENCES}

1. Johansson, M. A., Vasconcelos, P. F. \& Staples, J. E. The whole iceberg: estimating the incidence of yellow fever virus infection from the number of severe cases. Trans. R. Soc. Trop. Med. Hyg. 108, 482-487 (2014).

2. Ho, Y. L. et al. Severe yellow fever in Brazil: clinical characteristics and management. J. Travel Med. 26, https://doi.org/10.1093/jtm/taz040 (2019).

3. Rezende, I. M. et al. Persistence of Yellow fever virus outside the Amazon Basin, causing epidemics in Southeast Brazil, from 2016 to 2018. PLoS Negl. Trop. Dis. 12, e0006538 (2018)

4. Pan American Health Organization. Epidemiologic Update: Yellow Fever. https://www. paho.org/hq/index.php?option=com_docman\&view=download\&category_slug= yellow-fever-2194\&alias=43619-16-february-2018-yellow-fever-epidemiologicalupdate-619\&ltemid $=270 \&$ lang $=$ en (2018).

5. Moussallem, T. M. et al. Yellow fever outbreak in a rural-urban mixed community of Espirito Santo, Brazil: epidemiological aspects. Rev. Panam. salud publ. = Pan Am. J. Public Health 43, e29 (2019).

6. Shearer, F. M. et al. Global yellow fever vaccination coverage from 1970 to 2016: an adjusted retrospective analysis. Lancet Infect. Dis. 17, 1209-1217 (2017).

7. Barrett, A. D. Yellow fever vaccines. Biologicals 25, 17-25 (1997).

8. Staples, J. E., Monath, T. P., Gershman, M. D. \& Barrett, A. D. T. In Plotkin's Vaccines (eds Plotkin, S. A., Orenstein, W. A., Offit, P. A. \& Edwards, K. M.) 1181-1265 (Elsevier Inc., 2018).
9. Casey, R. M. et al. Immunogenicity of fractional-dose vaccine during a yellow fever outbreak-final report. N. Engl. J. Med. 381, 444-454 (2019).

10. Advisory Committee on Immunization Practices (ACIP). Grading of Recommendations, Assessment, Development, And Evaluation (GRADE) for Use of Yellow Fever Vaccine Booster Doses. https://www.cdc.gov/vaccines/acip/recs/grade/yf-vacboost.html (2015).

11. Mason, R. A., Tauraso, N. M., Spertzel, R. O. \& Ginn, R. K. Yellow fever vaccine: direct challenge of monkeys given graded doses of 17D vaccine. Appl. Microbiol. 25, 539-544 (1973).

12. Staples, J. E., Gershman, M. \& Fischer, M. Centers for Disease Control \& Prevention Yellow fever vaccine: recommendations of the Advisory Committee on Immunization Practices (ACIP).Morb. Mortal. Wkly. Rep. 59(RR07), 1-27 (2010).

13. Elliott, M. Yellow fever in the recently inoculated. Trans. R. Soc. Trop. Med. Hyg. $\mathbf{3 8}$ 231-234 (1944).

14. Courtois, G. [Duration of immunity after yellow fever vaccination]. Ann. Soc. Belg. Med. trop. 34, 9-12 (1954).

15. Dick, G. W. \& Gee, F. L. Immunity to yellow fever nine years after vaccination with 17D vaccine. Trans. R. Soc. Trop. Med. Hyg. 46, 449-458 (1952).

16. Groot, H. \& Riberiro, R. B. Neutralizing and haemagglutination-inhibiting antibodies to yellow fever 17 years after vaccination with 17D vaccine. Bull. World Health Organ. 27, 699-707 (1962).

17. Rosenzweig, E. C., Babione, R. W. \& Wisseman, C. L. Jr. Immunological studies with group B arthropod-borne viruses. IV. Persistence of yellow fever antibodies following vaccination with 17D strain yellow fever vaccine. Am. J. Trop. Med. Hyg. 12, 230-235 (1963).

18. Gotuzzo, E., Yactayo, S. \& Cordova, E. Efficacy and duration of immunity after yellow fever vaccination: systematic review on the need for a booster every 10 years. Am. J. Trop. Med. Hyg. 89, 434-444 (2013).

19. World Health Organization. SAGE Yellow fever work. Background Paper Yellow Fever Vaccines. http://www.who.int/entity/immunization/sage/meetings/2013/ april /1_Background_Paper_Yellow_Fever_Vaccines.pdf?ua=1 (2013).

20. World Health Organization. Vaccines and vaccination against yellow fever. WHO position paper-June 2013. Wkly. Epidemiol. Rec. 88, 269-283 (2013).

21. World Health Organization. International and Traveler Health: World-Yellow Fever Vaccination Booster. http://www.who.int/ith/updates/20140605/en/ (2014).

22. Staples, J. E., Bocchini, J. A. Jr., Rubin, L. \& Fischer, M. Centers for Disease Control and Prevention (CDC). Yellow fever vaccine booster doses: recommendations of the Advisory Committee on Immunization Practices, 2015. Morbid. Mortal. Wkly. Rep. 64, 647-650 (2015).

23. Grobusch, M. P. et al. Yellow fever revaccination guidelines change-a decision too feverish? Clin. Microbiol. Infect. 19, 885-886 (2013).

24. Plotkin, S. A. Ten yearly yellow fever booster vaccinations may still be justified. $J$. Travel Med. 25, https://doi.org/10.1093/jtm/tay130 (2018). 
25. Vasconcelos, P. F. Single shot of 17D vaccine may not confer life-long protection against yellow fever. Mem. Inst. Oswaldo Cruz 113, 135-137 (2018).

26. Amanna, I. J. \& Slifka, M. K. Questions regarding the safety and duration of immunity following live yellow fever vaccination. Expert Rev. Vaccines 15, 1519-1533 (2016).

27. Kareko, B. W. et al. Persistence of neutralizing antibody responses among yellow fever virus $17 \mathrm{D}$ vaccinees living in a nonendemic setting. J. Infect. Dis. 221, 2018-2025 (2020).

28. Collaborative group for studies on yellow fever. Duration of post-vaccination immunity against yellow fever in adults. Vaccine 32, 4977-4984 (2014).

29. Collaborative group for studies on yellow fever. Duration of immunity in recipients of two doses of 17DD yellow fever vaccine. Vaccine 37, 5129-5135 (2019).

30. Campi-Azevedo, A. C. et al. 17DD yellow fever revaccination and heightened long-term immunity in populations of disease-endemic areas, Brazil. Emerg. Infect. Dis. 25, 1511-1521 (2019).

31. Miyaji, K. T. et al. Prevalence and titers of yellow fever virus neutralizing antibodies in previously vaccinated adults. Rev. Inst. Med. Trop. Sao Paulo 59, e2 (2017).

32. de Menezes Martins, R. et al. Duration of post-vaccination immunity to yellow fever in volunteers eight years after a dose-response study. Vaccine 36, 4112-4117 (2018).

33. de Noronha, T. G. et al. Duration of post-vaccination humoral immunity against yellow fever in children. Vaccine 37, 7147-7154 (2019).

34. Domingo, C. et al. Long-term immunity against yellow fever in children vaccinated during infancy: a longitudinal cohort study. Lancet Infect. Dis. 19, 1363-1370 (2019).

35. Barrett, A. D. T. Yellow fever vaccine: the conundrum of 2 doses, one dose, or onefifth dose to induce and maintain protective immunity. J. Infect. Dis. 221, 1922-1924 (2020).

36. World Health Organization. Guidelines for Plaque Reduction Neutralization Testing of Human Antibodies to Dengue Viruses. https://apps.who.int/iris/bitstream/ handle/10665/69687/who_ivb_07.07_eng.pdf;jsessionid=19BF765D9B3FDAD85 EF3F217B0165953? sequence=1 (2007).

37. Hombach, J., Solomon, T., Kurane, I., Jacobson, J. \& Wood, D. Report on a WHO consultation on immunological endpoints for evaluation of new Japanese encephalitis vaccines, WHO, Geneva, 2-3 September, 2004. Vaccine 23, 5205-5211 (2005).

38. Roehrig, J. T., Hombach, J. \& Barrett, A. D. Guidelines for plaque-reduction neutralization testing of human antibodies to dengue viruses. Viral Immunol. 21, 123-132 (2008).

39. Johansson, M. A., Arana-Vizcarrondo, N., Biggerstaff, B. J. \& Staples, J. E. Incubation periods of Yellow fever virus. Am. J. Trop. Med. Hyg. 83, 183-188 (2010).

40. Visser, L. G., Veit, O. \& Chen, L. H. Waning immunity after single-dose yellow fever vaccination: who needs a second shot? J. Travel Med. 26, https://doi.org/10.1093/ jtm/tay134 (2019).

41. Kennedy, R. B., Lane, J. M., Henderson, D. A. \& Poland, G. A. in Plotkin's Vaccine (eds Plotkin, S. A., Orenstein, W. A., Offit, P. A. \& Edwards, K. M.) 1001-1030 (Elsevier Inc., 2018).

42. Erdman, D. D., Heath, J. L., Watson, J. C., Markowitz, L. E. \& Bellini, W. J. Immunoglobulin $\mathrm{M}$ antibody response to measles virus following primary and secondary vaccination and natural virus infection. J. Med. Virol. 41, 44-48 (1993).

43. Hepburn, M. J. et al. Neutralizing antibody response to booster vaccination with the 17d yellow fever vaccine. Vaccine 24, 2843-2849 (2006).

44. Brasil Ministerio da Saude. [Yellow Fever Seasonal Monitoring Brasil-2017/2018] https://portalarquivos2.saude.gov.br/images/pdf/2018/outubro/08/Informe-FA pdf (2018).

45. Camara, F. P., de Carvalho, L. M. \& Gomes, A. L. Demographic profile of sylvatic yellow fever in Brazil from 1973 to 2008. Trans. R. Soc. Trop. Med. Hyg. 107, 324-327 (2013)

46. Pan American Health Organization. Yellow Fever: Vaccination Status Among Confirmed Cases in the Americas, 2000-2014. http://ais.paho.org/phip/viz/ ed_yellowfever.asp (2019).

47. Minas Gerais Secretaria de Estado de Saude. [Epidemiologic Information on Yellow Fever]. http://www.saude.mg.gov.br/fornecimento-de-medicamentos/story/10284informe-epidemiologico-da-febre-amarela-27-02 (2018).

48. Tuboi, S. H., Costa, Z. G., da Costa Vasconcelos, P. F. \& Hatch, D. Clinical and epidemiological characteristics of yellow fever in Brazil: analysis of reported cases 1998-2002. Trans. R. Soc. Trop. Med. Hyg. 101, 169-175 (2007).

49. Filippis, A. M. et al. Isolation and characterization of wild type yellow fever virus in cases temporally associated with 17DD vaccination during an outbreak of yellow fever in Brazil. Vaccine 22, 1073-1078 (2004).

50. Saraiva, M. et al. Historical analysis of the records of sylvan yellow fever in the State of Amazonas, Brazil, from 1996 to 2009. Rev. Soc. Bras. Med. Trop. 46, 223-226 (2013).
51. Fereguetti, T. Novel clinical findings associated with YF in rural Brazil in Symposium 69: American Committee on Arthropod-Borne Viruses (ACAV) Symposium II: Everything Old Is New Again - The Re-Emergence of Yellow Fever. 68th Annual Meeting of American Society of Tropical Medicine and Hygiene (National Harbor, MD, USA, 2019).

52. Gibney, K. B. et al. Detection of anti-yellow fever virus immunoglobulin $\mathrm{m}$ antibodies at 3-4 years following yellow fever vaccination. Am. J. Trop. Med. Hyg. 87, 1112-1115 (2012).

53. Ferguson, M. Collaborative study to assess the suitability of a candidate International Standard for yellow fever vaccine. Biologicals 32, 195-205 (2004).

54. Wieten, R. W. et al. A single 17D yellow fever vaccination provides lifelong immunity; characterization of yellow-fever-specific neutralizing antibody and Tcell responses after vaccination. PLOS ONE 11, e0149871 (2016).

55. Campi-Azevedo, A. C. et al. Short-lived immunity after 17DD yellow fever single dose indicates that booster vaccination may be required to guarantee protective immunity in child. Front. Immunol. 10, 2192 (2019).

56. Lindsey, N. P. et al. Persistence of yellow fever virus-specific neutralizing antibodies after vaccination among US travellers. J. Travel Med. 25, https://doi.org/ 10.1093/jtm/tay108 (2018).

57. Roukens, A. H. E., van Halem, K., de Visser, A. W. \& Visser, L. G. Long-term protection after fractional-dose yellow fever vaccination: follow-up study of a randomized, controlled, noninferiority trial. Ann. Intern. Med. 169, 761-765 (2018).

58. Lopez, P. et al. Immunogenicity and safety of yellow fever vaccine (stamaril) when administered concomitantly with a tetravalent dengue vaccine candidate in healthy toddlers at 12-13 months of age in Colombia and Peru: a randomized trial. Pediatr. Infect. Dis. J. 35, 1140-1147 (2016).

59. Roy Chowdhury, P. et al. Immunogenicity of yellow fever vaccine coadministered with MenAfriVac in healthy infants in Ghana and Mali. Clin. Infect. Dis. 61, S586-593 (2015).

60. Idoko, O. T. et al. Antibody responses to yellow fever vaccine in 9 to 11-monthold Malian and Ghanaian children. Expert Rev. Vaccines 18, 867-875 (2019).

61. Muyanja, E. et al. Immune activation alters cellular and humoral responses to yellow fever 17D vaccine. J. Clin. Investig. 124, 3147-3158 (2014).

62. Albrecht, P., Ennis, F. A., Saltzman, E. J. \& Krugman, S. Persistence of maternal antibody in infants beyond 12 months: mechanism of measles vaccine failure. $J$. Pediatr. 91, 715-718 (1977).

63. Vasconcelos, P. F. C. \& Barrett, A. D. T. Are booster doses of yellow fever vaccine needed?. Lancet Infect. Dis. 19, 1275-1276 (2019).

64. Domingo, C. \& Niedrig, M. Safety of 17D derived yellow fever vaccines. Expert Opin. Drug Saf. 8, 211-221 (2009).

65. Kongsgaard, M. et al. Adaptive immune responses to booster vaccination against yellow fever virus are much reduced compared to those after primary vaccination. Sci. Rep. 7, 662 (2017).

66. Berkley, S. Health security's blind spot. Science 359, 1075 (2018).

67. Adrien, N. et al. Differences between coverage of yellow fever vaccine and the first dose of measles-containing vaccine: a desk review of global data sources. Vaccine 37, 4511-4517 (2019).

68. Wieten, R. W. et al. 17D yellow fever vaccine elicits comparable long-term immune responses in healthy individuals and immune-compromised patients. J. Infect. 72, 713-722 (2016).

69. Martins, R. M. et al. 17DD yellow fever vaccine: a double blind, randomized clinical trial of immunogenicity and safety on a dose-response study. Hum. Vaccines Immunother. 9, 879-888 (2013).

\section{ACKNOWLEDGEMENTS}

The findings and conclusions in this report are those of the authors and do not necessarily represent the official position of the Centers for Disease Control and Prevention and World Health Organization.

\section{AUTHOR CONTRIBUTIONS}

J.E.S., A.D.T.B., and J.H. contributed to the conception of the manuscript; J.E.S., A.D.T.B., A.W.-S., and J.H. contributed to reviewing and interpreting available literature; J.E.S., A.D.T.B., and A.W.-S. contributed to drafting the manuscript; and J.E.S., A.D.T.B., A.W.-S., and J.H. contributed to reviewing and editing the manuscript. All authors approved the submitted version and agreed both to be personally accountable for the author's own contributions and to ensure that questions related to the accuracy or integrity of any part of the work.

\section{COMPETING INTERESTS}

J.E.S., A.W.-S., J.H. declares no competing interests. A.D.T.B. is Editor-in-Chief of npj Vaccines. 


\section{ADDITIONAL INFORMATION}

Correspondence and requests for materials should be addressed to J.E.S.

Reprints and permission information is available at http://www.nature.com/ reprints

Publisher's note Springer Nature remains neutral with regard to jurisdictional claims in published maps and institutional affiliations.

Open Access This article is licensed under a Creative Commons Attribution 4.0 International License, which permits use, sharing, adaptation, distribution and reproduction in any medium or format, as long as you give appropriate credit to the original author(s) and the source, provide a link to the Creative Commons license, and indicate if changes were made. The images or other third party material in this article are included in the article's Creative Commons license, unless indicated otherwise in a credit line to the material. If material is not included in the article's Creative Commons license and your intended use is not permitted by statutory regulation or exceeds the permitted use, you will need to obtain permission directly from the copyright holder. To view a copy of this license, visit http://creativecommons. org/licenses/by/4.0/.

This is a U.S. government work and not under copyright protection in the U.S.; foreign copyright protection may apply 2020 